\title{
Preparing for the COVID-19 Mental Health Crisis in Latin America-Using Early Evidence from Countries that Experienced COVID-19 First
}

\author{
Alberto Abad1, Juliana Almeida da Silva2,3*, João Vitor Zamana das Neves Braga4, \\ Priscila Medeiros $2,3,5$, Renato Leonardo de Freitas $2,3,5,6$, Norberto Cysne Coimbra $2,3,7$, \\ José Aparecido da Silva ${ }^{*}$ \\ ${ }^{1}$ Department of Psychology, Federal University of Juiz de Fora (UFJF), Juiz de Fora, Brazil \\ ${ }^{2}$ Laboratory of Neuroanatomy and Neuropsychobiology, Department of Pharmacology, Ribeirão Preto Medical School of the \\ University of São Paulo (FMRP-USP), Ribeirão Preto, Brazil \\ ${ }^{3}$ Behavioural Neurosciences Institute (INeC), Ribeirão Preto, Brazil \\ ${ }^{4}$ State University of Santa Catarina, Florianópolis, Brazil \\ ${ }^{5}$ Laboratory of Neurosciences of Pain \& Emotions and Multi-User Centre of Neuroelectrophysiology, Department of Surgery and \\ Anatomy, Ribeirão Preto Medical School of the University of São Paulo, Ribeirão Preto, Brazil. \\ ${ }^{6}$ Biomedical Sciences Institute, Federal University of Alfenas (UNIFAL), Alfenas, Brazil \\ ${ }^{7}$ NAP-USP-Neurobiology of Emotions Research Centre (NuPNE), Ribeirão Preto Medical School of the University of São Paulo \\ (FMRP-USP), Ribeirão Preto, Brazil \\ ${ }^{8}$ Laboratory of Psychophysics, Perception, Psychometrics, and Pain, University of São Paulo at Ribeirão Preto, Ribeirão Preto, Brazil \\ Email: alberto.abad@ich.ufjf.br, *ju.silva@usp.br, *jadsilva@ffclrp.usp.br
}

How to cite this paper: Abad, A., da Silva, J.A., das Neves Braga, J.V.Z., Medeiros, P., de Freitas, R.L., Coimbra, N.C. and da Silva, J.A. (2020) Preparing for the COVID-19 Mental Health Crisis in Latin AmericaUsing Early Evidence from Countries that Experienced COVID-19 First. Advances in Infectious Diseases, 10, 40-44. https://doi.org/10.4236/aid.2020.103005

Received: May 1, 2020

Accepted: June 5, 2020

Published: June 8, 2020

Copyright () 2020 by author(s) and Scientific Research Publishing Inc. This work is licensed under the Creative Commons Attribution International License (CC BY 4.0).

http://creativecommons.org/licenses/by/4.0/

\begin{abstract}
The COVID-19 started in China and took over the world becoming a pandemic, arriving in Latin America in mid-February 2020. The South American continent is going through a delicate political, economic, and social moment that reflects the fragility of our health systems and science, contagion rates reduction strategies, and health services overload. Nevertheless, several studies are being carried out in Brazil and worldwide aiming to understand the characteristics of the disease and the cure of people infected by the coronavirus. Therefore, we must consider science-based strategies to combat the pandemic and promote a health system that takes care of the population.
\end{abstract}

\section{Keywords}

Mental Health, COVID-19, Psychological Reactions

\section{Introduction and Discussion}

With the spread of COVID-19 becoming a relevant public health problem in 
China on January 2020 and subsequently developing into a pandemic, as it spread to Iran, Europe, and North, Central and South Americas, approximately one billion people in China began to face various restrictions due to varying degrees of confinement. These included bans on public transportation, commerce, and business in general, which were then followed by quarantines on travelers returning from abroad, thus restricting most people from working and circulating normally. COVID-19 outbreak disrupted lives, work, triggered public panic, and mental health distress in China [1] hurting not only physically but also psychologically.

After China reported the first death from COVID-19 on January 11, new cases were confirmed in Thailand, Nepal, Australia, Malaysia, Singapore, South Korea, Vietnam, and Taiwan (China), gradually spreading out to countries like Iran, Italy, France, and the USA. As of May the $21^{\text {st }}$, the disease has infected more than five million people globally and killed over 325,000.

On February 26, Brazil announced the first case of COVID-19, and at the very moment we wrote this letter, the State of São Paulo has recorded 69,859 cases and 5363 deaths-more than China's total deaths. The disease has spread to every country in Latin America. Confirmed cases in Brazil have surpassed 293,000 with 18,894 deaths, although experts consider the actual number could be significantly higher given political issues, testing rates, and self-isolation levels: many scholars have stated that the ideal ratio for self-isolation in Brazil would be of $50 \%$ of the population. For the State of São Paulo, the most populous in the nation, an ideal isolation rate would be $70 \%$, nevertheless, these levels have never been achieved.

Indeed, regardless of the nation, there has never been a lockdown or quarantine of this magnitude, involving millions of people with no end in sight. This fact alone already gives the issue an emotional dimension that affects the stability of a population's mental health. We now have evidence of mental health issues in China [2] [3] and Iran [4].

Self-isolation is certainly an important "procedure" for safeguarding public health by stopping the propagation of the virus. However, it's also certain that the longer one spends in isolation, the bigger the chance of being afflicted by psychiatric, psychological, or cognitive disorders such as mood swings, depression, irritability, anxiety, fear, anger, insomnia, changes in appetite or subjective wellbeing. Pondering the role which social isolation might have had on the health of adults who had been subjected to the government-mandated, month-long lockdown in China, Dr. Stephen Zhang, from the University of Adelaide, Australia, along with his team, conducted a study [5] identifying adults participants, with pre-existing health conditions and unable to work due to the lockdown, as being the most vulnerable to suffering declines in their physical and mental health. To do so, the overall health of 369 adults, living in 64 distinct Chinese cities, with different rates of confirmed coronavirus cases were analyzed after each of the participants filled out a cross-transversal questionnaire.

Individual health was assessed employing a psychometric tool known as Physi- 
cal and Mental Health Survey (or SF-12). This survey has been translated and adapted, and its psychometric properties have been investigated in various intercultural studies. It contains twelve items and eight domains, which are: physical functioning ( 2 items), physical role ( 2 items), body pain ( 1 item), general health (1 item), vitality (1 item), social functioning ( 1 item), emotional role (2 items), and mental health (2 items). The eight domains, or factors, when combined, make up composite scales of mental and physical health with scores ranging between 0 and 100. When putting together, these results form a final SF12 score, in which higher numbers represent better health.

The Kessler Psychological Distress Scale (K6) was used to measured psychological stress, which has also been translated and adapted for several cultures. The scale contains six items which when submitted to a factorial analysis allow the extraction of two factors: anxiety and depression, representing different domains of psychological distress.

Lastly, the researchers measured an indicator of contentment with life utilizing the Satisfaction with Life Scale, globally renowned for its simplicity to use, validity, and accuracy. The scale was designed to measure global cognitive judgments of satisfaction with one's life (not to be confused with positive and negative affects). In this survey, the participants indicate how much they agree or disagree with each of the five items; utilizing numerical scores ranging from 7 (strongly agree) to 1 (strongly disagree).

Of all the participants, $27 \%$ worked in offices, $38 \%$ worked at home, and $25 \%$ had stopped working due to the outbreak of the virus. The categorical data reveals that those who stopped working showed a decrease in their physical and mental conditions, as well as the greatest levels of stress. Additionally, the severity of the disease in their city predicted their satisfaction with life, related contingently with pre-existing health issues and hours dedicated to physical exercise. In other words, adults who stopped working due to the outbreak saw a decline in both physical and mental health as well as an increase in stress.

Predictors of distress, during the COVID-19 pandemic, varies across countries due to medical systems, policies of lockdown, culture, labor and employment conditions, the information in both mainstream and social media, etc. [6]. Therefore, pandemic readiness and strategies coping with the disease differ across regions. In that sense, the World Health Organization (WHO) uses four methods to manage the spread of the infection (risk communication, vaccines and antiviral therapies, hygiene practices, and social distancing) [7].

In most Latin-American countries, healthcare systems are not prepared for the pandemic due to limitations in financing capacities, health infrastructure, equipment and health professionals shortage, thus increasing the risk of a destructive outbreak. Additionally, hygiene practices (washing hands with soap, using alcohol gel, sneezing in the fold of the arm, avoiding touching eyes, nose, and mouth, using face masks, putting tissues straight into a bin, etc.) and social distancing are not fully followed, especially in countries like Brazil and Mexico. 
Furthermore, due to the lack of scientifically supported vaccines and antiviral therapies, mass and indiscriminate testing, followed by high social distancing levels have been predictors of successfully curbing the spread of infection in countries like Germany and South Korea. Unfortunately, in other countries (Italy, England, France, Mexico, Brazil, etc.), due to their testing capacity, it has been limited to hospitals and patients with severe symptoms.

Treatment strategies of COVID-19 in Latin America differ from country to country based on their resources, expertise, and politics: several leaders have not seriously implemented policies. Brazil and Mexico presidents have been criticized not only for downplaying COVID-19 but also for ignoring the isolation strategies recommended by the World Health Organization (WHO) - therefore, politics is one of the biggest challenges for COVID-19 prevention in the region. Furthermore, the impact of cultural, political, and socioeconomic factors should be measured around the world and specifically in Latin-American countries. Consequences of COVID-19 are expected to impact unemployment, quality of work, and specific, more vulnerable groups as migrants, as stated by the International Labor Organization [8].

In the words of Doctor Zhang, the data show the need to monitor the health of those who were not infected by the virus, especially those who stopped working due to the lockdown. The authors also stress that physically active people may be more susceptible to changes in subjective well-being during this period. Therefore, public health policies should consider introducing restrictive measures to contain COVID-19, which can lead to an understanding of the implications to both the health and the subjective well-being of the people.

Zhang and his collaborators, therefore, state that identifying those which might be affected the most by COVID-19, not physically, but rather as a result of working and living in regions or states affected by the virus, will bring about important ramifications to public policies. This particularly applies to the gigantic country of Brazil. Identifying people in this group might help health organizations in prioritizing individuals in greater need, and consequently, health professionals, such as psychiatrists, psychologists, nurses, social workers, and even specialized volunteers, can be focused to provide services directed at the mental issues which may arise (or if already present, become more aggravated) during periods of isolation of any kind.

\section{Conclusions}

Finally, Zhang et al. suggest four recommendations for future interventions: having special attention to the most vulnerable groups (the elderly, women and migrant workers); improving the health service system and accessibility to medical resources; coordinating a psychological nationwide first aid plan during the COVID-19 pandemic; and implementing comprehensive crisis prevention and intervention system (epidemiological monitoring, screening, referral, and targeted intervention). 
Therefore, considering the conclusions emanating from the study by Zhang and collaborators as robust evidence, we strongly recommend them to be implemented as public policies in Latin America. It is of utmost importance to facilitate indiscriminate testing among citizens, achieve social distancing levels recommended by the WHO, and define specific treatment and COVID-19 recovery strategies based on the characteristics of each country.

\section{Authors' Contributions}

Abad prepared and wrote of the manuscript; Juliana Almeida da Silva, wrote and revised the manuscript; J.V.Z. Neves Braga prepared and wrote the manuscript; P. Medeiros revised the manuscript; R.L. de Freitas revised the manuscript; N.C. Coimbra revised the manuscript, and J.A. da Silva wrote and revised the manuscript.

\section{Conflicts of Interest}

The authors declare no conflicts of interest regarding the publication of this paper.

\section{References}

[1] Bao, Y., Sun, Y., Meng, S., Shi, J. and Lu, L. (2020) COVID-19 Epidemic: Address Mental Health Care to Empower Society. The Lancet, 395, e37-e38. https://doi.org/10.1016/S0140-6736(20)30309-3

[2] Qiu, J., Shen, B., Zhao, M., Wang, Z., Xie, B. and Xu, Y. (2020) A Nationwide Survey of Psychological Distress among Chinese People in the COVID-19 Epidemic: Implications and Policy Recommendations. General Psychiatry, 33, e100213. https://doi.org/10.1136/gpsych-2020-100213

[3] Li, S., Wang, Y., Xue, J., Zhao, N. and Zhu, T. (2020) The Impact of COVID-19 Epidemic Declaration on Psychological Consequences: A Study on Active Weibo Users. International Journal of Environmental Research and Public Health, 17, 2032. https://doi.org/10.3390/ijerph17062032

[4] Jahanshahi, A.A., Dinani, M.M., Madavani, A.N., Li, J. and Zhang, S.X. (2020) The Distress of Iranian Adults during the Covid-19 Pandemic-More Distressed than the Chinese and with Different Predictors. medRxiv. https://doi.org/10.1016/j.bbi.2020.04.081

[5] Zhang, S.X., Wang, Y., Rauch, A. and Wei, F. (2020) Unprecedented Disruption of Lives and Work: Health, Distress and Life Satisfaction of Working Adults in China One Month into the COVID-19 Outbreak. Psychiatry Research, 112958. https://doi.org/10.1016/j.psychres.2020.112958

[6] Zandifar, A. and Badrfam, R. (2020) Iranian Mental Health during the COVID-19 Epidemic. Asian Journal of Psychiatry, 51. https://doi.org/10.1016/j.ajp.2020.101990

[7] World Health Organization (2008) WHO Outbreak Communication Planning Guide. Geneva.

[8] International Labour Organization (2020) COVID-19 and the World of Work: Impact and Policy Responses. 